Archive for

Organic Chemistry

Arkivoc 2019, part v, 60-72

\title{
Simple synthesis of 2-alkylidene- and 2-keto-7-triazolylquinoxaline systems from 2-nitrosodiarylamines
}

\author{
Zbigniew Wróbel, ${ }^{*}$ Robert Bujok, Michał Tryniszewski, and Andrzej Kwast \\ Institute of Organic Chemistry, Polish Academy of Sciences, ul. Kasprzaka 44, 01-224 Warsaw, Poland \\ Email: zbigniew.wrobel@icho.edu.pl
}

Received 09-28-2018

Accepted 01-04-2019

Published on line $02-15-2019$

\section{Abstract}

A synthesis of triazole-substituted 1-arylquinoxaline derivatives from halogenated 2-nitrosodiarylamines by two routes is presented. Regioselective substitution of fluorine or chlorine in the starting compounds by sodium azide followed by double cyclocondensation of both the azide substituent and the nitrosoamine group leads to functionalized 2-methylenequinoxaline derivatives. The alternative route separates the two cyclocondensation reactions, allowing obtention of triazole-substituted quinoxalin-2-one derivatives by using two different dicarbonyl reagents.

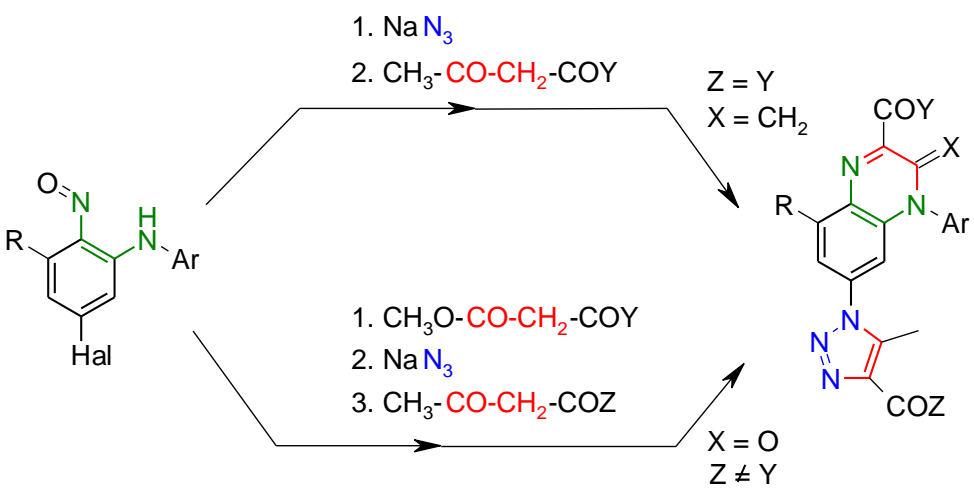

Keywords: Triazoles, cyclocondensation, heterocycles, nucleophilic aromatic substitution, nitroso group 


\section{Introduction}

Triazoles are compounds of great importance particularly in medicinal chemistry. ${ }^{1-4}$ Nitrogen polycyclic heterocycles possessing triazole ring as a substituent attract continuous attention. Synthesis of triazoles is accomplished most frequently using the azido group as a three-nitrogen atom donor. Thus, heteroaromatic azides become crucial starting materials in the synthesis of such polyheterocyclic systems.

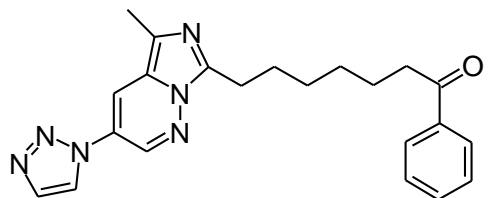

A

anti-HIV activity

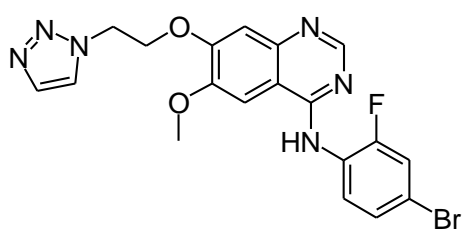

B

VEGF receptor tyrosine kinase inhibitor

Figure 1. Examples of important structures of polyheterocyclic compounds $\mathbf{A}^{5}$ and $\mathbf{B}^{6}$ bearing triazole ring.

In 2007, we discovered the reaction of nitroarenes with aromatic amines in the presence of a strong base leading to 2-nitrosodiarylamines ${ }^{7,8}$ These compounds turned out to be versatile starting materials for synthesis of a number of two-nitrogen-containing heterocycles such as phenazines, ${ }^{9,10}$ benzimidazoles ${ }^{11}$ and quinoxalinones. ${ }^{12}$ On the other hand, when the leaving group was present in the ring containing the nitroso group, the latter acted as a strong electron-withdrawing group activating the leaving group for aromatic nucleophilic substitution. The reaction was found efficient for alkoxy or alkylamine nucleophiles. However, due to high reactivity of the nitroso group towards various nucleophiles as well as its susceptibility to redox processes, the choice of potential nucleophiles has been limited. ${ }^{13}$

Recently we found that the range of suitable nucleophiles can be extended to azide anions. In this report, we present a simple synthesis of 5-azido-2-nitrosodiarylamines which can be further transformed into 2alkylidenequinoxalines bearing triazole group in position 7 , via the cascade reaction with $\beta$-dicarbonyl compounds. This method allows for obtaining complex structures starting from simple substrates under mild, heavy-metal-free conditions. Furthermore, a complementary approach which make use of 2nitrosodiarylamines in the synthesis of triazole-substituted quinoxalin-2-one derivatives is presented.

\section{Results and Discussion}

Substitution of halogens $(\mathrm{F}, \mathrm{Cl})$ at the para position to the nitroso group with sodium azide proceeded smoothly with very good to excellent yields at room temperature in the systems: water-alcohol, dipolar aprotic solvents or catalytic two-phase solid-liquid system (PTC). PTC was chosen for a standard procedure (Table 1). The obtained crude products were pure enough for further use, thus, as they are not stable under column chromatographic conditions, they were used without purification.

The reaction was highly regioselective. Azide ions exclusively substituted the para-chloro position in 3,5dichloro-2-nitrosodiarylamine (Table 1, entry 7). This is in accordance with the results of the previously examined reactions of halogenated nitrosodiarylamines with alkyl amines or alcohols. ${ }^{13}$ The regioselectivity 
has been attributed to the significant contribution of the quinoid structure in the substrate, which is a result of the conjugation between the nitroso and the ortho-arylamine group. ${ }^{13}$

Table 1. Substitution of halogens in 2-nitrosodiarylamines 1 by azide ions

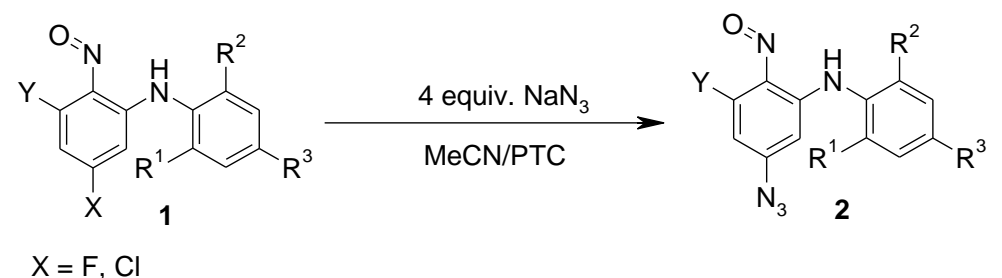

\begin{tabular}{|c|c|c|c|c|c|c|c|c|c|}
\hline \multirow{2}{*}{ Entry } & \multicolumn{3}{|c|}{ Nitrosodiarylamine } & \multirow{2}{*}{$\mathrm{R}^{1}$} & \multirow{2}{*}{$\mathrm{R}^{2}$} & \multirow{2}{*}{$\mathrm{R}^{3}$} & \multicolumn{3}{|c|}{ Azide $\mathbf{2}$} \\
\hline & $x$ & $\mathrm{Y}$ & 1 & & & & 2 & Conditions $^{a}$ & Yield $^{\mathrm{b}} \%$ \\
\hline 1 & $\mathrm{~F}$ & $\mathrm{H}$ & 1a & I & $\mathrm{H}$ & $\mathrm{Cl}$ & $2 a$ & A & $71^{\mathrm{c}}$ \\
\hline 2 & $\mathrm{~F}$ & $\mathrm{H}$ & $1 b$ & Me & Me & $\mathrm{H}$ & $2 b$ & $A$ & 99 \\
\hline 3 & $\mathrm{Cl}$ & $\mathrm{H}$ & 1c & I & $\mathrm{H}$ & $\mathrm{Me}$ & $2 c$ & B & 98 \\
\hline 4 & $\mathrm{Cl}$ & $\mathrm{H}$ & $1 d$ & $t-\mathrm{Bu}$ & $\mathrm{H}$ & $\mathrm{H}$ & $2 d$ & B & 96 \\
\hline 5 & $\mathrm{Cl}$ & $\mathrm{H}$ & $1 e$ & Me & Me & Me & $2 e$ & B & 97 \\
\hline 6 & $\mathrm{Cl}$ & $\mathrm{H}$ & $1 f$ & Et & Me & $\mathrm{H}$ & $2 f$ & B & 89 \\
\hline 7 & $\mathrm{Cl}$ & $\mathrm{Cl}$ & $1 g$ & $\mathrm{Me}$ & Me & $\mathrm{H}$ & $2 g$ & B & $98 / 63^{c}$ \\
\hline
\end{tabular}

${ }^{a}$ Conditions A: MeCN 5\% mol Bu ${ }_{4} \mathrm{NBr}$; B: $\mathrm{MeCN}, 10 \% \mathrm{~mol} \mathrm{Bu}_{4} \mathrm{NHSO}_{4}$.

${ }^{\mathrm{b}}$ Crude products, pure enough for further transformations.

${ }^{c}$ Isolated and purified by column chromatography.

Substitution of ortho-chloride, when it is the only leaving group in the molecule, occurs very slowly and is followed by intramolecular condensation of the azido and nitroso groups resulted in formation of a benzofurazan system 4 a (Scheme 2). The ortho azido nitroso compounds $\mathbf{3}$ were not isolated nor observed, but it seems reasonable to assume them as intermediates leading to 4 . Surprisingly, the methoxy group at para position resists of substitution by azide ions in $\mathbf{1} \mathbf{i}$, while it was preferentially substituted with pyrrolidine in 3-chloro-5-methoxy-2-nitrosodiarylamines. ${ }^{13}$

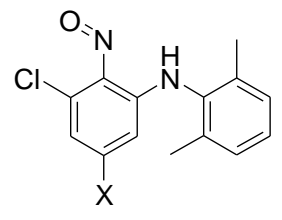

1h $\mathrm{X}=\mathrm{H}$ 1i $X=O M e$

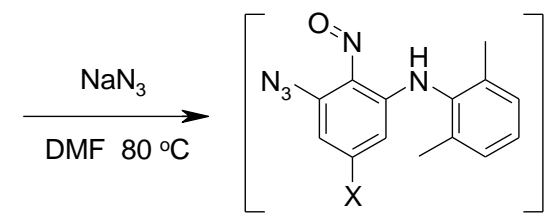

3

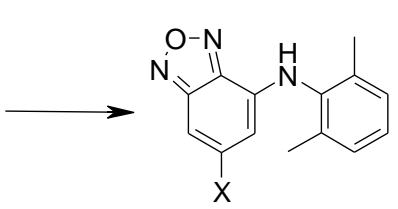

4a $\mathrm{X}=\mathrm{H} \quad 77 \%$ yield 4b $X=$ OMe $76 \%$ yield

Scheme 1. Formation of arylaminobenzofurazans from 2-nitrosodiarylamines $\mathbf{1 h}, \mathbf{1 i}$ and sodium azide.

The substitution of ortho halogens by azide ions forming benzofurazan derivatives was observed and described earlier for a few 2,6-dihalonitrosobenzenes in their reaction with sodium azide in DMSO at elevated 
temperature. ${ }^{14,15}$ Since the cyclization seems to be much faster than the substitution, obtaining $o$ azidonitrosoarenes this way was not possible.

Functionalization of 2-nitrosodiarylamines with an azido group opened up a new perspective for building complex heterocyclic systems containing multiple rings. Initially we tried to build a triazole ring by coppercatalyzed cycloaddition ${ }^{16}$ of the azido group with propargyl alcohol derivatives but the results were disappointing, probably because of reactivity of the nitroso group under the reaction conditions. Thus, we turned out to the most straightforward and reasonable idea to build two different heterocyclic rings on the two reactive centers, the ortho-nitrosoaniline and the azido functions, in the reaction with the same shared reagent. While double condensation reactions of $\mathbf{2}$ with benzyl cyanide, cyanoacetic acid esters and malonates were unsuccessful, with 1,3-diketones or $\beta$-ketoesters gave positive results. The reaction worked satisfactorily in $\mathrm{MeCN}$ with various $\beta$-diketones, provided that they possessed at least one terminal methyl group. tertButylamine was chosen as a catalyst since it had been found to be the best catalyst for the previously developed reaction of 2-nitrosodiarylamines with $\beta$-diketones leading to the condensed pyrazine ring. ${ }^{17,18}$

Table 2. Double cyclization of $\mathbf{2}$ with 1,3-dicarbonyl compounds
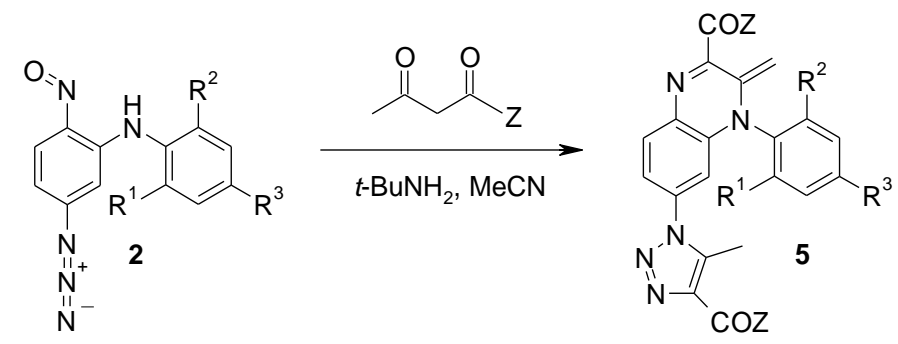

\begin{tabular}{ccccc}
\hline \multirow{2}{*}{ Entry } & Azide $^{\mathrm{a}}$ & \multicolumn{3}{c}{ Double cyclization } \\
\cline { 2 - 5 } & $\mathbf{2}$ & Z & $\mathbf{5}$ & Yield $^{\mathrm{b}}(\%)$ \\
\hline 2 & $\mathbf{2 a}$ & Me & $\mathbf{5 a}$ & 49 \\
3 & $\mathbf{2 b}$ & $\mathrm{Me}$ & $\mathbf{5 b}$ & 82 \\
4 & $\mathbf{2 b}$ & $\mathrm{OEt}$ & $\mathbf{5 c}$ & 22 \\
5 & $\mathbf{2 b}$ & $i-\mathrm{Pr}$ & $\mathbf{5 d}$ & 54 \\
6 & $\mathbf{2 c}$ & $\mathrm{Me}$ & $\mathbf{5 e}$ & 56 \\
7 & $\mathbf{2 d}$ & $\mathrm{Me}$ & $\mathbf{5 f}$ & 51 \\
8 & $\mathbf{2 e}$ & $\mathrm{Me}$ & $\mathbf{5 g}$ & 65 \\
9 & $\mathbf{2 e}$ & $\mathrm{iPr}$ & $\mathbf{5 h}$ & 40 \\
10 & $\mathbf{2 f}$ & $\mathrm{Me}$ & $\mathbf{5 i}$ & 71 \\
11 & $\mathbf{2 f}$ & $i-P r$ & $\mathbf{5 j}$ & 46 \\
\hline
\end{tabular}

a Substituents as shown in Table 1.

${ }^{\mathrm{b}}$ Isolated yields.

Formation of the triazole ring proceeds most likely via the dipolar cycloaddition of the enolate form of $\beta$ dicarbonyl compound and aryl azide, ${ }^{19-21}$ for which detailed mechanistic studies and explanation of the observed regioselectivity have been reported. ${ }^{19}$ The reactions $\mathbf{2}>\mathbf{5}$ are also regioselective. The expected structure of the regioisomer formed was confirmed for selected triazoles (5a, $\mathbf{d}$ and $\mathbf{f})$ by NMR techniques 
HMBC and NOESY. The latter proved proximity of the triazole methyl group and appropriate protons of the dihydroquinoxaline aromatic ring.

Our efforts to perform consecutively both cyclizations failed, i.e. by using different dicarbonyl reagents with $\mathrm{NO}$ and $\mathrm{N}_{3}$ groups. This was probably because of a multistep character of both cyclizations, and because they take place in distant regions of the starting molecule. Hence, they may occur independently of each other at the same time. Consecutive, controlled formation of both heterocyclic rings, using different dicarbonyl reagents dedicated for particular cyclization, required a different approach. Thus, we tried to introduce the azido function into bicyclic systems obtained by condensation of 2-nitrosodiarylamines with dicarbonyl compounds. This condensation, which has been described previously, ${ }^{11,12}$ allows for the formation of quinoxalin-2-one derivatives possessing alkoxycarbonyl substituent at C3. Carbocyclic rings of these compounds seem to be electrophilic enough for the nucleophilic substitution of halogens, placed in the activated positions 5 or 7, with azide ions. Thus, further condensation of the bicyclic azides could be carried out with another carbonyl reagent. As an illustration of this approach, the three-step synthetic sequence starting from representative 2-nitrosodiarylamines was accomplished (Table 3).

Table 3. Separate cyclocondensations of the nitrosodiarylamine moiety and the azido group

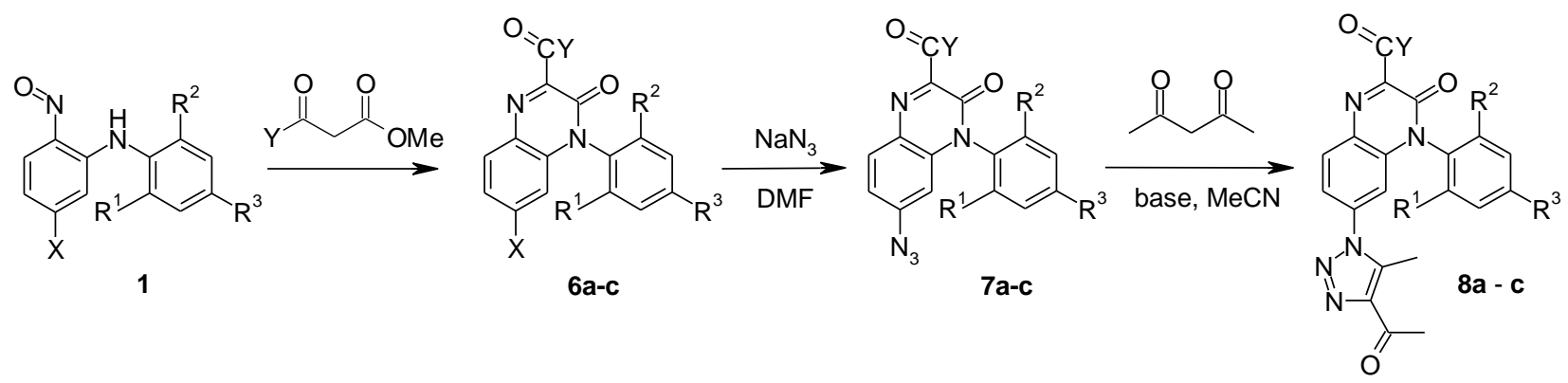

\begin{tabular}{cccccccccccc}
\hline Entry & $\mathrm{X}$ & $\mathrm{R}^{1}$ & $\mathrm{R}^{2}$ & $\mathrm{R}^{3}$ & $\mathrm{Y}$ & \multicolumn{2}{c}{ 6 Yield $^{\mathrm{a}}$ (\%) } & 7 Yield $^{\mathrm{a}}$ (\%) & 8 Yield $^{\mathrm{a}}(\%)$ \\
\hline 1 & $\mathrm{~F}$ & $\mathrm{Me}$ & $\mathrm{Me}$ & $\mathrm{H}$ & $\mathrm{MeO}$ & $\mathbf{6 a}$ & 81 & $\mathbf{7 a}$ & 70 & $\mathbf{8 a}$ & 46 \\
2 & $\mathrm{~F}$ & $\mathrm{H}$ & $\mathrm{H}$ & $\mathrm{Cl}$ & $\mathrm{Ph}$ & $\mathbf{6 b}$ & 60 & $\mathbf{7 b}$ & 80 & $\mathbf{8 b}$ & 81 \\
3 & $\mathrm{Cl}$ & $\mathrm{H}$ & $\mathrm{H}$ & $\mathrm{Me}$ & $\mathrm{MeO}$ & $\mathbf{6 c}$ & 64 & $\mathbf{7 c}$ & 37 & $\mathbf{8 c}$ & 81 \\
\hline
\end{tabular}

asolated yields.

While fluorine was substituted by sodium azide in quinoxalin-2-ones $\mathbf{6 a}$ and $\mathbf{6} \mathbf{b}$ efficiently at room temperature, the reaction of chloro derivative $6 \mathrm{c}$ required elevated temperature $\left(80^{\circ} \mathrm{C}\right)$ and gave much lower yield of 7c. On the other hand, relatively low yield of the cyclization of $7 a$ is difficult to explain. Nevertheless, the approach can be useful for the synthesis of complex nitrogen heterocyclic compounds of a specific structure.

\section{Conclusions}

It was demonstrated that halogenated 2-nitrosodiarylamines can be useful starting materials in the synthesis of triazole-substituted 1-arylquinoxaline systems by two routes. The two-step method benefits from the 
efficient and regioselective nucleophilic substitution of 5-fluorine or 5-chlorine in 2-nitrosodiarylamines by sodium azide and is followed by double condensation with acetylketones. Some limitations of the method can be circumvented by separation of the two cyclization reactions. Thus, in the alternative approach, 7halogenoquinoxalinones are prepared first, followed by substitution of halogens to form the intermediate azidoquinoxalinones. This allows to use two different dicarbonyl compounds for both cyclization processes, and therefore extends the scope of tricyclic systems obtained from 2-nitrosodiarylamines.

\section{Experimental Section}

General. ${ }^{1} \mathrm{H}$ and ${ }^{13} \mathrm{C}$ NMR spectra were recorded on a Varian-NMR-vnmrs600 and a Varian Mercury 500 instruments at $298 \mathrm{~K}$. Chemical shifts are expressed in ppm referred to TMS ( $\left.{ }^{1} \mathrm{H} N M R\right)$ or to the solvent used $\left({ }^{13} \mathrm{C} N M R\right)$, with coupling constants in Hertz. Mass spectra were obtained on an AutoSpec Premier (Waters) spectrometer (EI, $70 \mathrm{eV}$ ) and an API 365i spectrometer (ESI in MeOH). Silica gel Merck 60 (230-400 mesh) was used for column chromatography. THF was distilled from sodium/benzophenone ketyl prior to use. DMF was dried over $\mathrm{CaH}_{2}$, distilled and stored over molecular sieves. Common reagents and materials were purchased from commercial sources and used as received. Preparation and characterization of 2-nitrosodiarylamines 1, except 1a, $\mathbf{1 e}$ and $\mathbf{1}$, have been described in our previous papers. ${ }^{8,17,18}$ Quinoxalinones 6a-c were prepared following the procedure published earlier. ${ }^{12}$

$\mathbf{N}$-(5-Fluoro-2-nitrosophenyl)- $\mathbf{N}$-(4-chloro-2-iodophenyl)amine (1a). To a solution of $t$-BuOK (3.4 g, $30 \mathrm{mmol})$ in dry THF $(50 \mathrm{~mL})$ cooled to $-74{ }^{\circ} \mathrm{C}$ was added 4-chloro-2-iodoaniline $(7.6 \mathrm{~g}, 30 \mathrm{mmol})$ in THF $(5 \mathrm{~mL})$ then a solution of 4-fluoronitrobenzene $(4.23 \mathrm{~g}, 30 \mathrm{mmol})$ in THF $(5 \mathrm{~mL})$ was added dropwise. The mixture was stirred at $\mathrm{ca}-70{ }^{\circ} \mathrm{C}$ for $60 \mathrm{~min}$ then the mixture was alowed to warm up slowly to $-30{ }^{\circ} \mathrm{C}$, then poured into conc. $\mathrm{HCl}_{\mathrm{aq}}$ $(20 \mathrm{~mL})$ in water $(400 \mathrm{~mL})$ and extracted with EtOAc. The extract was washed with water and dried $\left(\mathrm{Na}_{2} \mathrm{SO}_{4}\right)$. After evaporation the product was isolated by column chromatography $\left(\mathrm{SiO}_{2}\right.$, hexane/toluene 2:1). Pure 1a (5.75 g, 51\%) was obtained as a brown solid. $\mathrm{mp} 148{ }^{\circ} \mathrm{C}$ (dec.). ${ }^{1} \mathrm{H} \mathrm{NMR}\left(500 \mathrm{MHz}, \mathrm{CDCl}_{3}\right): \delta 6.45-6.49(\mathrm{~m}, 1 \mathrm{H})$, 7.75-6.81 (m, 1H), $7.31(\mathrm{~d}, J 8.5 \mathrm{~Hz}, 1 \mathrm{H}), 7.40(\mathrm{dd}, J$ 8.5, $2.2 \mathrm{~Hz}, 1 \mathrm{H}), 7.94(\mathrm{~d}, J 2.2 \mathrm{~Hz}, 1 \mathrm{H}), 8.76(\mathrm{br}, 1 \mathrm{H}), 11.58$ (br s, $1 \mathrm{H}) ;{ }^{13} \mathrm{C} \mathrm{NMR}\left(125 \mathrm{MHz}, \mathrm{CDCl}_{3}\right): \delta 96.4,99.8$ (d, $\left.J_{\mathrm{CF}} 25 \mathrm{~Hz}\right), 107.9$ (d, $\left.J_{\mathrm{CF}} 27 \mathrm{~Hz}\right), 126.6,129.6,132.9,137.8$, 139.5, 154.8, 168.0 (d, $J_{\text {CF }} 262 \mathrm{~Hz}$ ) (two signal missing); ${ }^{22}$ HRMS (ESI): $\mathrm{m} / \mathrm{z}$ calcd for $\mathrm{C}_{12} \mathrm{H}_{7}{ }^{35} \mathrm{CIFIN}_{2} \mathrm{ONa}$ : 398.9173, found: 398.9167

$\mathbf{N}$-(5-Chloro-2-nitrosophenyl)- $\mathbf{N}$-mesitylamine (1e). To a cooled to $-74{ }^{\circ} \mathrm{C}$ solution of $t$-BuOK $(3.4 \mathrm{~g}, 30 \mathrm{mmol})$ in dry THF $(50 \mathrm{~mL})$ was added mesitylamine $(1.3 \mathrm{~g}, 9.6 \mathrm{mmol})$ in THF $(5 \mathrm{~mL})$ then a solution of 4chloronitrobenzene $(1.51 \mathrm{~g}, 9.6 \mathrm{mmol})$ in THF $(5 \mathrm{~mL})$ was added dropwise. The mixture was stirred at ca $-70{ }^{\circ} \mathrm{C}$ for 40 min then poured into saturated $\mathrm{NH}_{4} \mathrm{Cl}$ and extracted with EtOAc. The extract was washed with water and dried $\left(\mathrm{Na}_{2} \mathrm{SO}_{4}\right)$. After evaporation the product was isolated by column chromatography $\left(\mathrm{SiO}_{2}\right.$, hexane/toluene 5:1 - 1:1. Pure 1 e $(2.08 \mathrm{~g}, 78 \%)$ was obtained as a dark solid. mp 64-69 ${ }^{\circ} \mathrm{C} .{ }^{1} \mathrm{H} \mathrm{NMR}(500 \mathrm{MHz}$, $\mathrm{CDCl}_{3}$ ): $\delta 2.05(\mathrm{~s}, 6 \mathrm{H}), 2.32(\mathrm{~s}, 3 \mathrm{H}), 6.32(\mathrm{~d}, J 1.7 \mathrm{~Hz}, 1 \mathrm{H}), 6.90(\mathrm{br} \mathrm{d}, J 8.2 \mathrm{~Hz}, 1 \mathrm{H}), 6.97(\mathrm{~s}, 2 \mathrm{H}), 8.75(\mathrm{br} s, 1 \mathrm{H})$, 11.71 (br s, $1 \mathrm{H}) ;{ }^{13} \mathrm{C} \mathrm{NMR}\left(125 \mathrm{MHz}_{\mathrm{CDCl}}\right.$ ): $\delta$ 18.0, 21.0, 114.2, 118.1, 129.5, 130.5, 135.5, 137.9, 142.1, 144.8, 155.1 (one signal missing); ${ }^{22} \mathrm{MS}(\mathrm{EI}): \mathrm{m} / z(\%) 274\left(\mathrm{M}^{+}, 1\right), 261$ (46), 259 (100), 242 (21); HRMS (EI): $\mathrm{m} / z$ calcd for $\mathrm{C}_{15} \mathrm{H}_{15}{ }^{35} \mathrm{Cl} \mathrm{N}$ O: 274.0873, found: 274.0872 .

$\mathbf{N}$-(5-Chloro-2-nitrosophenyl)-N-(2-ethyl-6-methylphenyl)amine (1f). Obtained according to the procedure described for 1e from 2-ethyl-6-methylaniline $(1.30 \mathrm{~g}, 9.6 \mathrm{mmol})$ and 4-chloronitrobenzene $(1.51 \mathrm{~g}, 9.6 \mathrm{mmol})$ 
as dark oil (2.03 g, 77\%). ${ }^{1} \mathrm{H}$ NMR $\left(600 \mathrm{MHz}, \mathrm{CDCl}_{3}\right): \delta 1.09(\mathrm{t}, J 7.5 \mathrm{~Hz}, 3 \mathrm{H}), 2.08(\mathrm{~s}, 3 \mathrm{H}), 2.43(\mathrm{q}, J 7.5 \mathrm{~Hz}, 2 \mathrm{H})$, $6.30(\mathrm{~d}, J 1.1 \mathrm{~Hz}, 1 \mathrm{H}), 6.91(\mathrm{~d}, J 7.3 \mathrm{~Hz}, 1 \mathrm{H}), 7.16(\mathrm{~d}, J 7.6 \mathrm{~Hz}, 1 \mathrm{H}), 7.18(\mathrm{~d}, J 7.6 \mathrm{~Hz}, 1 \mathrm{H}), 7.25(\mathrm{t}, J 7.6 \mathrm{~Hz}, 1 \mathrm{H})$, 8.78 (br s, 1H), 11.81 (br s, $1 \mathrm{H}) ;{ }^{13} \mathrm{C}$ NMR (150 MHz, $\mathrm{CDCl}_{3}$ ): $\delta$ 14.7, 18.1, 24.8, 114.2, 118.2, 127.1, 128.4, $128.8,132.5,136.0,141.7,142.2,144.8,155.0$ (one signal missing); ${ }^{22} \mathrm{MS}$ (EI): $\mathrm{m} / z(\%) 274\left(\mathrm{M}^{+}, 2\right), 259(36)$, 245 (100); HRMS (ESI): $m / z$ calcd for $\mathrm{C}_{15} \mathrm{H}_{16}{ }^{35} \mathrm{CIN}_{2} \mathrm{O}[\mathrm{M}+\mathrm{H}]^{+}: 275.0951$, found: 275.0947.

General procedure for the reactions of 2-nitrosodiarylamines 1a-g with sodium azide. A mixture of 2-nitrosodiarylamine $1(0.5 \mathrm{mmol})$, sodium azide $(129 \mathrm{mg}, 2.0 \mathrm{mmol})$ and $\mathrm{NBu}_{4} \mathrm{HSO}_{4}(69 \mathrm{mg}, 0.2 \mathrm{mmol})$ in $\mathrm{CH}_{3} \mathrm{CN}(4 \mathrm{~mL}$ ) was stirred at room temperature for 2-5 h (tlc controlled). When the reaction was complete $\mathrm{CH}_{3} \mathrm{CN}$ was evaporated at room temperature. To the residue $\mathrm{Et}_{2} \mathrm{O}(40 \mathrm{~mL})$ and water $(5 \mathrm{~mL})$ were added and the layers were separated. The organic layer was dried $\left(\mathrm{Na}_{2} \mathrm{SO}_{4}\right)$ and the solvent was evaporated to obtain products pure enough for further reactions. When it was necessary, the $\mathrm{Et}_{2} \mathrm{O}$ solution was poured through a small $\mathrm{SiO}_{2}$ pad before evaporation to remove tars. Additional purification for analytical purposes was performed by column chromatography $\left(\mathrm{SiO}_{2}\right.$, hexane/ethyl acetate, 9:1 to 2:1). and/or recrystallization from hexane/ethyl acetate mixture.

5-Azido-4'-chloro-2'-iodo-2-nitrosodiphenylamine (2a). Brown powder (142 mg, 71\%). mp $127-129{ }^{\circ} \mathrm{C} .{ }^{1} \mathrm{H}$ NMR $\left(500 \mathrm{MHz}, \mathrm{CDCl}_{3}\right): \delta 6.35(\mathrm{~s}, 1 \mathrm{H}), 6.75$ (br d, J $\left.8.3 \mathrm{~Hz}, 1 \mathrm{H}\right), 7.29(\mathrm{~d}, J 8.3 \mathrm{~Hz}, 1 \mathrm{H}), 7.39(\mathrm{dd}, J 8.3,2.2 \mathrm{~Hz}$, 1H), 7.93 (d, J $2.2 \mathrm{~Hz}, 1 \mathrm{H}), 8.67$ (br s, 1H), 11.65 (br s, 1H). ${ }^{13} \mathrm{C} \mathrm{NMR} \mathrm{(125} \mathrm{MHz,} \mathrm{CDCl} 3$ ): $\delta$ 96.4, 102.8, 110.1, 126.6, 129.5, 132.8, 137.9, 139.6, 149.7, 154.6 (two signals invisible); ${ }^{22} \mathrm{MS}$ (EI): $\mathrm{m} / z$ (\%) 399 (4), 246 (100), 227 (76). HRMS (EI): $m / z$ calcd for $\mathrm{C}_{12} \mathrm{H}_{7} \mathrm{~N}_{5}{ }^{35} \mathrm{CllO}$ : 398.9384, found: 398.9391 .

5-Azido-2',6'-dimethyl-2-nitrosodiphenylamine (2b). Dark crystals (133 mg, 71\%). mp 98-102 ${ }^{\circ} \mathrm{C}\left(\right.$ dec.); ${ }^{1} \mathrm{H}$ NMR $\left(500 \mathrm{MHz}, \mathrm{CDCl}_{3}\right): \delta 2.10(\mathrm{~s}, 6 \mathrm{H}), 5.80(\mathrm{~s}, 1 \mathrm{H}), 6.67$ (br s, $\left.1 \mathrm{H}\right), 7.12-7.17(\mathrm{~m}, 2 \mathrm{H}), 7.18-7.22(\mathrm{~m}, 1 \mathrm{H}), 8.80$ (br s, 1H), 11.99 (br s, 1H). ${ }^{13} \mathrm{C}$ NMR $\left(125 \mathrm{MHz}, \mathrm{CDCl}_{3}\right): \delta$ 18.1, 102.6, 109.1, 121.0, 128.1, 128.8, 135.8, 143.4, 149.5, 154.7, (one signal invisible); ${ }^{22} \mathrm{MS}(\mathrm{EI}): \mathrm{m} / z$ (\%) 267 (19), 252 (100), 224 (92), 207 (86), 144 (66); HRMS (EI): $m / z$ calcd for $\mathrm{C}_{14} \mathrm{H}_{13} \mathrm{~N}_{5} \mathrm{O}: 267.1120$, found: 267.1112 .

5-Azido-2'-iodo-4'-methyl-2-nitrosodiphenylamine (2c). Dark red oil (185 mg, 98\%); ${ }^{1} \mathrm{H} \mathrm{NMR}(500 \mathrm{MHz}$, $\left.\mathrm{CDCl}_{3}\right): \delta 2.35(\mathrm{~s}, 3 \mathrm{H}), 6.32(\mathrm{~s}, 1 \mathrm{H}), 6.66-6.73(\mathrm{~m}, 1 \mathrm{H}), 7.15-7.26(\mathrm{~m}, 2 \mathrm{H}), 7.78(\mathrm{~s}, 1 \mathrm{H}), 8.76(\mathrm{br} \mathrm{s}, 1 \mathrm{H}), 11.87(\mathrm{br}$ s, $1 \mathrm{H}) .{ }^{13} \mathrm{C} N M R\left(125 \mathrm{MHz}, \mathrm{CDCl}_{3}\right): \delta 20.5,96.4,109.8,114.4,118.9,126.3,130.1,136.1,139.1,140.6,144.6$, 154.6, 155.0; MS (ESI): $m / z$ (\%) $402[\mathrm{M}+\mathrm{Na}]^{+}$, HRMS (ESI): $\mathrm{m} / z$ calcd for $\mathrm{C}_{13} \mathrm{H}_{10} \mathrm{~N}_{5} \mathrm{IONa}$ : 401.9228, found: 401.9217.

5-Azido-2'-t-butyl-2-nitrosodiphenylamine (2d). Brown solid (150 mg, 96\%). mp $96{ }^{\circ} \mathrm{C}$ (dec.); ${ }^{1} \mathrm{H} \mathrm{NMR}(500$ $\mathrm{MHz}_{\mathrm{CDCl}}$ ): $\delta 1.37(\mathrm{~s}, 9 \mathrm{H}), 6.24$ (br s, $\left.1 \mathrm{H}\right), 6.67$ (br s, $\left.1 \mathrm{H}\right), 7.14-7.18(\mathrm{~m}, 1 \mathrm{H}), 7.23-7.27(\mathrm{~m}, 1 \mathrm{H}), 7.29(\mathrm{ddd}, J$ 7.7, 7.4, $1.5 \mathrm{~Hz}, 1 \mathrm{H}), 7.52$ (dd J 7.7, $1.5 \mathrm{~Hz}, 1 \mathrm{H}), 8.76$ (br s, $1 \mathrm{H}), 12.58$ (br s, $1 \mathrm{H}) ;{ }^{13} \mathrm{C} \mathrm{NMR}\left(125 \mathrm{MHz} \mathrm{CDCl}_{3}\right): \delta$ 30.6, 35.0, 103.3, 109.4, 114.2, 127.1, 127.6, 127.7, 127.8, 129.3, 134.6, 136.3, 143.1, 146.3, 149.5, 154.9; MS (EI): $m / z$ (\%) 295 (2), 250 (92), 238 (74), 210 (100), 194 (74); MS (ESI): $\mathrm{m} / z 318$ [M+Na] ; HRMS (ESI): $\mathrm{m} / z$ calcd for $\mathrm{C}_{16} \mathrm{H}_{17} \mathrm{~N}_{5} \mathrm{ONa}$ : 318.1331, found: 318.1315 .

5-Azido-2-nitroso-2', 4',6'-trimethyldiphenylamine (2e). Dark oil (136 mg, 97\%). mp 93-96 ${ }^{\circ} \mathrm{C} .{ }^{1} \mathrm{H}$ NMR (500 $\mathrm{MHz}_{\mathrm{CDCl}}$ ): $\delta 2.05(\mathrm{~s}, 6 \mathrm{H}), 2.32(\mathrm{~s}, 3 \mathrm{H}), 5.81(\mathrm{~s}, 1 \mathrm{H}), 6.66$ (br s, 1H), $6.95(\mathrm{~s}, 2 \mathrm{H}), 8.78$ (br s, $\left.1 \mathrm{H}\right), 11.97$ (br s, $1 \mathrm{H}) ;{ }^{13} \mathrm{C}$ NMR $\left(125 \mathrm{MHz} \mathrm{CDCl}_{3}\right): \delta$ 18.0, 21.0, 102.6, 109.1, 129.4, 130.6, 135.3, 136.7, 137.8, 140.2, 149.5, 154.7; MS (ESI): $m / z$ (\%) 304 [M+Na] ${ }^{+}$, HRMS (ESI): $m / z$ calcd for $\mathrm{C}_{15} \mathrm{H}_{15} \mathrm{~N}_{5} \mathrm{ONa:} 304.1174$, found: 304.1167.

5-Azido-2'-ethyl-6'-methyl-2-nitrosodiphenylamine (2f). Brown solid (125 mg, 89\%); mp 130-132 ${ }^{\circ} \mathrm{C}\left(\mathrm{dec}\right.$ )); ${ }^{1} \mathrm{H}$ NMR $\left(500 \mathrm{MHz}_{\mathrm{CDCl}}\right.$ ): $\delta 1.10(\mathrm{t}, \mathrm{J} 7.5 \mathrm{~Hz}, 3 \mathrm{H}), 2.08(\mathrm{~s}, 3 \mathrm{H}), 2.40-2.46(\mathrm{~m}, 2 \mathrm{H}), 5.80(\mathrm{~s}, 1 \mathrm{H}), 6.67$ (br s, 1H), 7.14$7.19(\mathrm{~m}, 2 \mathrm{H}), 7.23-7.25(\mathrm{~m}, 1 \mathrm{H}), 8.80$ (br s, 1H), 12.06 (br s, $1 \mathrm{H}) .{ }^{13} \mathrm{C} \mathrm{NMR}\left(125 \mathrm{MHz}, \mathrm{CDCl}_{3}\right): \delta 14.8,18.1,24.8$, 
102.6, 109.1, 127.1, 128.3, 128.8, 132.7, 135.9, 136.6, 141.6, 143.2, 149.6, 154.6; MS (ESI): $\mathrm{m} / \mathrm{z} 280$ [M-H] ${ }^{+}$, HRMS (ESI): $m / z$ calcd for $\mathrm{C}_{15} \mathrm{H}_{14} \mathrm{~N}_{5} \mathrm{O}: 280.1998$, found: 280.1998 .

5-Azido-3-chloro-2', 6'-dimethyl-2-nitrosodiphenylamine (2g). Brown crystals, crude yield $148 \mathrm{mg}$, 98\%), after column chromatography (hexane/EtOAc) $95 \mathrm{mg}, 63 \%) . \mathrm{mp} 128-130{ }^{\circ} \mathrm{C} .{ }^{1} \mathrm{H} \mathrm{NMR}\left(500 \mathrm{MHz}, \mathrm{CDCl}_{3}\right): \delta 2.08(\mathrm{~s}$, $6 \mathrm{H}), 5.73(\mathrm{~d}, J 2.2 \mathrm{~Hz}, 1 \mathrm{H}), 6.74(\mathrm{~d}, J 2.2 \mathrm{~Hz}, 1 \mathrm{H}), 7.13-7.16(\mathrm{~m}, 2 \mathrm{H}), 7.19-7.23(\mathrm{~m}, 1 \mathrm{H}), 12.62(\mathrm{br} \mathrm{s}, 1 \mathrm{H}) .{ }^{13} \mathrm{C} \mathrm{NMR}$ $\left(125 \mathrm{MHz}, \mathrm{CDCl}_{3}\right): \delta$ 18.0, 101.9, 110.1, 128.4, 128.9, 132.9, 135.5, 137.6, 147.6, 150.1, 150.5; MS (El): $\mathrm{m} / \mathrm{z}$ (\%) 301 (73), 258 (74), 207 (82), 193 (100), 144 (95), HRMS (EI): $m / z$ calcd for $\mathrm{C}_{14} \mathrm{H}_{12}{ }^{35} \mathrm{CIN}_{5} \mathrm{O}: 301.0730$, found: 301.0723.

General procedure for the reactions of 3-chloro-2-nitrosodiarylamines $1 \mathrm{~h}$ and $\mathbf{1 i}$ with sodium azide. A mixture of 2-nitrosodiarylamine $1(0.5 \mathrm{mmol})$ and sodium azide $(129 \mathrm{mg}, 2.0 \mathrm{mmol})$ in DMF $(5 \mathrm{~mL})$ was stirred at room temperature for 10 days or for $24 \mathrm{~h}$ at $80{ }^{\circ} \mathrm{C}$ with comparable results. When the reaction was complete the mixture was diluted with water $(70 \mathrm{~mL})$ and extracted with EtOAc $(30 \mathrm{~mL})$. The extract was washed with water $(3 \times 50 \mathrm{~mL})$, brine and dried $\left(\mathrm{Na}_{2} \mathrm{SO}_{4}\right)$. The solvent was evaporated to obtain crude products which were purified by column chromatography $\left(\mathrm{SiO}_{2}\right.$, hexane/ethyl acetate, 9:1 to $\left.1: 1\right)$ then recrystallized from $i$-PrOH for analytical purposes.

N-(2,6-Dimethylphenyl)-2,1,3-benzoxadiazol-4-amine (4a). Yellow crystals, $92 \mathrm{mg}, 77 \%$ yield. $\mathrm{mp} 116-117{ }^{\circ} \mathrm{C}$ (i-PrOH); ${ }^{1} \mathrm{H}$ NMR $\left(500 \mathrm{MHz}, \mathrm{CDCl}_{3}\right): \delta 2.26(\mathrm{~s}, 6 \mathrm{H}), 5.72(\mathrm{~d}, J 7.1 \mathrm{~Hz}, 1 \mathrm{H}), 6.28(\mathrm{br} \mathrm{s}, 1 \mathrm{H}), 7.09(\mathrm{~d}, J 8.9 \mathrm{~Hz}, 1 \mathrm{H})$, 7.14 (dd, J 8.9, $7.1 \mathrm{~Hz}, 1 \mathrm{H}), 7.17-7.21(\mathrm{~m}, 3 \mathrm{H}) .{ }^{13} \mathrm{C} \mathrm{NMR}\left(125 \mathrm{MHz}, \mathrm{CDCl}_{3}\right): \delta 18.0,102.9,103.1,127.4,128.8$, 133.9, 134.5, 135.5, 136.4, 144.7, 150.2. MS (EI): $\mathrm{m} / z$ (\%) 239 (87), 224 (47), 194 (100). HRMS (EI): $\mathrm{m} / \mathrm{z}$ calcd for $\mathrm{C}_{14} \mathrm{H}_{13} \mathrm{~N}_{3} \mathrm{O}: 239.1059$, found: 239.1065 .

N-(2,6-Dimethylphenyl)-6-methoxy-2,1,3-benzoxadiazol-4-amine (4b). Yellow crystals, $103 \mathrm{mg}, 76 \%$ yield. mp $126-128{ }^{\circ} \mathrm{C}(\mathrm{i}-\mathrm{PrOH}) ;{ }^{1} \mathrm{H}$ NMR $\left(500 \mathrm{MHz}, \mathrm{CDCl}_{3}\right): \delta 2.55(\mathrm{~s}, 6 \mathrm{H}), 3.81(\mathrm{~s}, 3 \mathrm{H}), 5.43(\mathrm{~d}, J 1.9 \mathrm{~Hz}, 1 \mathrm{H}), 6.23(\mathrm{br} \mathrm{s}, 1 \mathrm{H})$, $6.26(\mathrm{~d}, J 1.9 \mathrm{~Hz}, 1 \mathrm{H}), 7.15-7.18(\mathrm{~m}, 3 \mathrm{H}) .{ }^{13} \mathrm{C} \mathrm{NMR}\left(125 \mathrm{MHz}, \mathrm{CDCl}_{3}\right): \delta 18.0,55.6,78.8,99.3,127.5,128.8$, 134.9, 135.2, 136.3, 143.3, 151.0, 164.6. MS (EI): $\mathrm{m} / \mathrm{z}$ (\%) 269 (100), 254 (41), 224 (90). HRMS (EI): $\mathrm{m} / \mathrm{z}$ calcd for $\mathrm{C}_{15} \mathrm{H}_{15} \mathrm{~N}_{3} \mathrm{O}: 269.1164$, found: 269.1173 .

General procedure for the double condensation of 5-azido-2-nitrosodiarylamines 2 with dicarbonyl compounds. A solution of $2(0.5 \mathrm{mmol})$, the dicarbonyl compound $(1.1 \mathrm{mmol})$ and $t-\mathrm{BuNH}_{2}(175 \mathrm{mg}, 2.4 \mathrm{mmol})$ in $\mathrm{CH}_{3} \mathrm{CN}(5.0 \mathrm{~mL})$ was stirred at room temperature for $24 \mathrm{~h}$. When the reaction was complete $\mathrm{CH}_{3} \mathrm{CN}$ was evaporated. Water $(4 \mathrm{~mL})$ was added to the residue and the mixture was extracted with EtOAc $(10 \mathrm{~mL})$. The extract was dried $\left(\mathrm{Na}_{2} \mathrm{SO}_{4}\right)$ and the solvent was evaporated. The crude product was purified by column chromatography $\left(\mathrm{SiO}_{2}\right.$, hexane/ethyl acetate, 9:1 to 2:1) to obtain pure product 5 .

1-\{1-[2-Acetyl-4-(4-chloro-2-iodophenyl)-3-methylene-3,4-dihydroquinoxalin-6-yl]-5-methyl-1H-1,2,3-triazol4-yl\}ethanone (5a). Dark solid (135 mg, 49\%). mp 93-96 ${ }^{\circ} \mathrm{C}$ (dec.); ${ }^{1} \mathrm{H} \mathrm{NMR}\left(500 \mathrm{MHz}, \mathrm{CDCl}_{3}\right): \delta 2.51(\mathrm{~s}, 3 \mathrm{H})$, $2.64(\mathrm{~s}, 3 \mathrm{H}), 2.69(\mathrm{~s}, 3 \mathrm{H}), 3.68(\mathrm{~d}, J 2.6 \mathrm{~Hz}, 1 \mathrm{H}), 4.99(\mathrm{~d}, J 2.6 \mathrm{~Hz}, 1 \mathrm{H}), 5.92(\mathrm{~d}, J 2.1 \mathrm{~Hz}, 1 \mathrm{H}), 6.93$ (dd, J 8.2, 2.1 $\mathrm{Hz}, 1 \mathrm{H}), 7.27(\mathrm{~d}, J 8.2 \mathrm{~Hz}, 1 \mathrm{H}), 7.57(\mathrm{~d}, J 2.3 \mathrm{~Hz}, 1 \mathrm{H}), 7.56-7.63(\mathrm{~m}, 2 \mathrm{H}) ;{ }^{13} \mathrm{CNMR}\left(125 \mathrm{MHz}, \mathrm{CDCl}_{3}\right): \delta 10.2,27.7$, 27.8, 92.8, 99.6, 108.8, 117.3, 131.1, 131.4, 132.0, 132.9, 134.5, 136.1, 136.4, 137.1, 137.3, 138.3, 141.5, 143.7, 156.6, 194.2, 198.7; MS (EI): $\mathrm{m} / \mathrm{z}$ (\%) 545 (21), 390 (17), 43 (100); HRMS (EI): $\mathrm{m} / \mathrm{z}$ calcd for $\mathrm{C}_{22} \mathrm{H}_{17}{ }^{35} \mathrm{ClIN}_{5} \mathrm{O}_{2}: 545.0116$, found: 545.0125 .

1-\{1-[2-Acetyl-4-(2,6-dimethylphenyl)-3-methylene-3,4-dihydroquinoxalin-6-yl]-5-methyl-1H-1,2,3-triazol-4yl\}ethanone (5b). Dark red solid (170 mg, 82\%). mp 128-133 ${ }^{\circ} \mathrm{C} .{ }^{1} \mathrm{H}$ NMR $\left(500 \mathrm{MHz}, \mathrm{CDCl}_{3}\right): \delta 2.13(\mathrm{~s}, 6 \mathrm{H}), 2.43$ (s, 3H), $2.64(\mathrm{~s}, 3 \mathrm{H}), 2.68(\mathrm{~s}, 3 \mathrm{H}), 3.65(\mathrm{~d}, J 1.9 \mathrm{~Hz}, 1 \mathrm{H}), 4.87$ (d, J $1.9 \mathrm{~Hz}, 1 \mathrm{H}), 5.86(\mathrm{~d}, J 2.0 \mathrm{~Hz}, 1 \mathrm{H}), 6.89$ (dd, J 
8.2, $2.0 \mathrm{~Hz}, 1 \mathrm{H}), 7.22-7.27(\mathrm{~m}, 3 \mathrm{H}), 7.56(\mathrm{~d}, J 8.2 \mathrm{~Hz}, 1 \mathrm{H}) ;{ }^{13} \mathrm{C} \mathrm{NMR}\left(125 \mathrm{MHz}, \mathrm{CDCl}_{3}\right): \delta 10.1,17.7,27.7,27.8$, 90.3, 107.7, 116.6, 129.5, 130.1, 131.0, 132.8, 133.8, 134.0, 136.4, 136.6, 137.1, 137.5, 143.7, 157.0, 194.2, 199.0; MS (EI): $m / z$ (\%) 413 (53), 370 (96), 342 (100); HRMS (EI): $m / z$ calcd for $\mathrm{C}_{24} \mathrm{H}_{23} \mathrm{~N}_{5} \mathrm{O}_{2}: 413.1852$, found: 413.1847.

Ethyl 6-[4-(ethoxycarbonyl)-5-methyl-1H-1,2,3-triazol-1-yl]-4-(2,6-dimethylphenyl)-3-methylene-3,4dihydroquinoxaline-2-carboxylate (5c). Brown solid $(52 \mathrm{mg}, 22 \%)$ mp 93-96 ${ }^{\circ} \mathrm{C} .{ }^{1} \mathrm{H} \mathrm{NMR}(500 \mathrm{MHz} \mathrm{CDCl}): \delta$ $1.40(\mathrm{t}, J 7.2 \mathrm{~Hz}, 3 \mathrm{H}), 1.44(\mathrm{t}, J 7.2 \mathrm{~Hz}, 3 \mathrm{H}), 2.14(\mathrm{~s}, 6 \mathrm{H}), 2.42(\mathrm{~s}, 3 \mathrm{H}), 3.64(\mathrm{~d}, J 2.4 \mathrm{~Hz}, 1 \mathrm{H}), 4.39-4.48(\mathrm{~m}, 5 \mathrm{H})$, $5.88(\mathrm{~d}, J 2.2 \mathrm{~Hz}, 1 \mathrm{H}), 6.91(\mathrm{dd}, J$ 8.3, $2.2 \mathrm{~Hz}, 1 \mathrm{H}), 7.23-7.28(\mathrm{~m}, 3 \mathrm{H}), 7.60(\mathrm{~d}, J 8.3 \mathrm{~Hz}, 1 \mathrm{H}) ;{ }^{13} \mathrm{C} \mathrm{NMR}(125 \mathrm{MHz}$, $\left.\mathrm{CDCl}_{3}\right): \delta 9.9,14.1,14.3,17.7,61.1,62.4,89.1,107.9,117.1,129.6,130.1,130.8,132.9,133.9,134.8,136.0$, 136.4, 136.8, 137.2, 138.6, 155.3, 161.6, 164.1; MS (EI): m/z (\%) 473 (70), 430 (100), 372 (74), 356 (57); HRMS (EI): $m / z$ calcd for $\mathrm{C}_{26} \mathrm{H}_{27} \mathrm{~N}_{5} \mathrm{O}_{4}: 473.2063$, found: 473.2061 .

1-\{1-[4-(2,6-Dimethylphenyl)-2-isobutyryl-3-methylene-3,4-dihydroquinoxalin-6-yl]-5-methyl-1H-1,2,3-

triazol-4-yl\}-2-methylpropan-1-one (5d). Red solid (130 mg, 54\%). mp 143-144 $\left.{ }^{\circ} \mathrm{C} .{ }^{1} \mathrm{H} \mathrm{NMR} \mathrm{(500} \mathrm{MHz} \mathrm{CDCl}_{3}\right)$ : $\delta 1.22(\mathrm{~d}, J 6.9 \mathrm{~Hz}, 6 \mathrm{H}), 1.24(\mathrm{~d}, J 6.9 \mathrm{~Hz}, 6 \mathrm{H}), 2.14(\mathrm{~s}, 6 \mathrm{H}), 2.44(\mathrm{~s}, 3 \mathrm{H}), 3.61(\mathrm{~d}, J 2.2 \mathrm{~Hz}, 1 \mathrm{H}), 3.64(\mathrm{q}, J 6.9 \mathrm{~Hz}$, $1 \mathrm{H}), 3.85(\mathrm{q}, J 6.9 \mathrm{~Hz}, 1 \mathrm{H}), 4.67(\mathrm{~d}, J 2.2 \mathrm{~Hz}, 1 \mathrm{H}), 5.91(\mathrm{~d}, J 2.0 \mathrm{~Hz}, 1 \mathrm{H}), 6.90(\mathrm{dd}, J 8.2,2.0 \mathrm{~Hz}, 1 \mathrm{H}), 7.22-7.90(\mathrm{~m}$, 3H), 7.54 (d, J $8.2 \mathrm{~Hz}, 1 \mathrm{H}) ;{ }^{13} \mathrm{C}$ NMR $\left(125 \mathrm{MHz}_{\mathrm{CDCl}}\right.$ ): $\delta 10.1,17.6,17.7,18.6,37.1,37.4,89.6,107.8,116.8$, $129.5,130.1,130.6,133.0,134.1,134.7,136.2,136.5,137.2,137.7,142.5,159.6,200.7,204.6 ; \mathrm{MS}$ (EI): $\mathrm{m} / z$ (\%) 469 (100), 440 (55), 370 (56), 300 (38); HRMS (EI): $m / z$ calcd for $\mathrm{C}_{28} \mathrm{H}_{31} \mathrm{~N}_{5} \mathrm{O}_{2}$ : 469.2478, found: 469.2483.

1-\{1-[2-Acetyl-4-(2-iodo-4-methylphenyl)-3-methylene-3,4-dihydroquinoxalin-6-yl]-5-methyl-1H-1,2,3-

triazol-4-yl\}ethanone (5e). Brown solid (147 mg, 56\%). mp 67-71 ${ }^{\circ} \mathrm{C} .{ }^{1} \mathrm{H} \mathrm{NMR}\left(600 \mathrm{MHz}, \mathrm{CDCl}_{3}\right): \delta 2.39(\mathrm{~s}, 3 \mathrm{H})$, $2.49(\mathrm{~s}, 3 \mathrm{H}), 2.63(\mathrm{~s}, 3 \mathrm{H}), 2.69(\mathrm{~s}, 3 \mathrm{H}), 3.68(\mathrm{~d}, J 2.4 \mathrm{~Hz}, 1 \mathrm{H}), 4.93(\mathrm{~d}, J 2.4 \mathrm{~Hz}, 1 \mathrm{H}), 5.88(\mathrm{~d}, J 2.2 \mathrm{~Hz}, 1 \mathrm{H}), 6.91$ (dd, J 8.3, 2.2 Hz, 1H), 7.16 (d, J $8.0 \mathrm{~Hz}, 1 \mathrm{H}), 7.36$ (ddd, J 8.0, 2.0, 0.7 Hz, 1H), 7.56 (d, J $8.3 \mathrm{~Hz}, 1 \mathrm{H}), 7.89$ (dd, J 2.0, $0.7 \mathrm{~Hz}, 1 \mathrm{H}) ;{ }^{13} \mathrm{C} \mathrm{NMR}\left(150 \mathrm{MHz}, \mathrm{CDCl}_{3}\right): \delta 10.2,20.7,26.9,27.7,92.3,98.6,109.0,116.9,130.0,130.9$, 132.4, 132.9, 134.8, 136.8, 136.9, 137.0, 137.3, 141.6, 142.3, 143.7, 157.0, 194.3, 198.9; MS (EI): $\mathrm{m} / z$ (\%) 525 (39), 370 (48), 302 (40), 43 (100); HRMS (EI): $\mathrm{m} / z$ calcd for $\mathrm{C}_{23} \mathrm{H}_{20} \mathrm{~N}_{5} \mathrm{IO}_{2}: 525.0662$, found: 525.0671.

1-\{1-[2-Acetyl-4-(2-t-butylphenyl)-3-methylene-3,4-dihydroquinoxalin-6-yl]-5-methyl-1H-1,2,3-triazol-4-

yl\}ethanone (5f). Brown solid (112 mg, 51\%). mp 89-91 ${ }^{\circ} \mathrm{C} .{ }^{1} \mathrm{H} N M R\left(500 \mathrm{MHz} \mathrm{CDCl}_{3}\right): \delta 1.34(\mathrm{~s}, 9 \mathrm{H}), 2.44(\mathrm{~s}$, $3 \mathrm{H}), 2.62(\mathrm{~s}, 3 \mathrm{H}), 2.68(\mathrm{~s}, 3 \mathrm{H}), 3.68(\mathrm{~d}, J 1.8 \mathrm{~Hz}, 1 \mathrm{H}), 4.97(\mathrm{~d}, J 1.8 \mathrm{~Hz}, 1 \mathrm{H}), 5.92(\mathrm{~d}, J 2.0 \mathrm{~Hz}, 1 \mathrm{H}), 6.86(\mathrm{dd}, J 8.2$, $2.0 \mathrm{~Hz}, 1 \mathrm{H}), 7.00(\mathrm{~d}, J 7.6 \mathrm{~Hz}, 1 \mathrm{H}), 7.36-7.45(\mathrm{~m}, 2 \mathrm{H}), 7.54(\mathrm{~d}, J 8.2,1 \mathrm{H}), 7.68(\mathrm{~d}, J 7.6 \mathrm{~Hz}, 1 \mathrm{H}) ;{ }^{13} \mathrm{C} \mathrm{NMR}(125$ $\mathrm{MHz}_{1} \mathrm{CDCl}_{3}$ ): $\delta$ 10.1, 27.7, 27.8, 31.8, 36.1, 94.0, 110.38, 116.6, 129.6, 129.7, 130.6, 131.1, 131.7, 133.2, 134.9, 136.9, 137.1, 137.5, 139.3, 143.6, 148.1, 156.9, 194.2, 198.9; MS (EI): $m / z$ (\%) 541 (33), 398 (100), 370 (55), 356 (52); HRMS (EI): $m / z$ calcd for $\mathrm{C}_{26} \mathrm{H}_{27} \mathrm{~N}_{5} \mathrm{O}_{2}: 441.2165$, found: 441.2161 .

1-\{1-[2-Acetyl-3-methylene-4-(2,4,6-trimethylphenyl) -3,4-dihydroquinoxalin-6-yl]-5-methyl-1H-1,2,3-triazol4-yl\}ethanone (5g). Dark solid (136 mg, 66\%). mp 95-99 ${ }^{\circ} \mathrm{C} .{ }^{1} \mathrm{H} N M R\left(500 \mathrm{MHz}, \mathrm{CDCl}_{3}\right): \delta 2.08(\mathrm{~s}, 6 \mathrm{H}), 2.32(\mathrm{~s}$, 3H), $2.44(\mathrm{~s}, 3 \mathrm{H}), 2.63(\mathrm{~s}, 3 \mathrm{H}), 2.69(\mathrm{~s}, 3 \mathrm{H}), 3.68(\mathrm{~d}, J 1.8 \mathrm{~Hz}, 1 \mathrm{H}), 4.84(\mathrm{~d}, J 1.8 \mathrm{~Hz}, 1 \mathrm{H}), 5.89(\mathrm{~d}, J 2.1 \mathrm{~Hz}, 1 \mathrm{H})$, $6.88(\mathrm{dd}, J$ 8.3, $2.1 \mathrm{~Hz}, 1 \mathrm{H}), 7.03(\mathrm{~s}, 2 \mathrm{H}), 7.54(\mathrm{~d}, J 8.3 \mathrm{~Hz}, 1 \mathrm{H}) ;{ }^{13} \mathrm{C} \mathrm{NMR}\left(125 \mathrm{MHz}, \mathrm{CDCl}_{3}\right): \delta$ 10.1, 17.6, 21.1, 26.9, 27.7, 27.8, 90.2, 107.9, 116.5, 130.8, 130.9, 131.3, 132.9, 134.0, 135.9, 136.9, 137.1, 137.5, 139.4, 143.7, 157.1, 194.3, 199.1; MS (EI): m/z (\%) 427 (56), 384 (83), 356 (87), 314 (37), 43 (100); HRMS (EI): $m / z$ calcd for $\mathrm{C}_{25} \mathrm{H}_{25} \mathrm{~N}_{5} \mathrm{O}_{2}: 427.2010$, found: 427.2008 .

1-\{1-[2-Isobutyryl-3-methylene-4-(2,4,6-trimethylphenyl)-3,4-dihydroquinoxalin-6-yl]-5-methyl-1H-1,2,3triazol-4-yl\}-2-methylpropan-1-one (5h). Brown solid (97 mg, 40\%). mp 166-170 ${ }^{\circ} \mathrm{C}$ (dec). ${ }^{1} \mathrm{H} \mathrm{NMR}(600 \mathrm{MHz}$, $\left.\mathrm{CDCl}_{3}\right): \delta 1.23(\mathrm{~d}, J 7.0 \mathrm{~Hz}, 6 \mathrm{H}), 1.24(\mathrm{~d}, J 7.0 \mathrm{~Hz}, 6 \mathrm{H}), 1.25(\mathrm{~s}, 3 \mathrm{H}), 2.09(\mathrm{~s}, 6 \mathrm{H}), 2.32(\mathrm{~s}, 3 \mathrm{H}), 2.45(\mathrm{~s}, 3 \mathrm{H}), 3.62(\mathrm{br}$ s, 1H), 3.63 (sept, J 7.0, 1H), 3.86 (sept, J 7.0, 1H), 4.44 (br s, 1H), 5.94 (d, J 2.2 Hz, 1H), 6.89 (dd, J 8.2, $2.2 \mathrm{~Hz}$, 
1H), 7.03 (s, 2H), 7.53 (d, J 8.2, 1H); $\left.{ }^{13} \mathrm{C} \mathrm{NMR} \mathrm{(150} \mathrm{MHz,} \mathrm{CDCl}_{3}\right): \delta 10.1,17.5,17.6,18.6,21.1,37.1,37.4,89.5$, 108.0, 116.6, 129.9, 130.5, 130.8, 131.4, 133.0, 136.0, 136.4, 137.1, 137.7, 139.4, 142.5, 159.6, 200.7, 204.7; MS (ESI): $m / z$ (\%) $484[M]^{+}$, HRMS (ESI): $m / z$ calcd for $\mathrm{C}_{29} \mathrm{H}_{34} \mathrm{~N}_{5} \mathrm{O}_{2}: 484.2713$, found: 484.2709 .

1-\{1-[2-Acetyl-4-(2-ethyl-6-methylphenyl)-3-methylene-3,4-dihydroquinoxalin-6-yl]-5-methyl-1H-1,2,3triazol-4-yl\}ethanone (5i). Dark solid (151 mg, 71\%). mp 94-98 $\left.{ }^{\circ} \mathrm{C} .{ }^{1} \mathrm{H} \mathrm{NMR} \mathrm{(500} \mathrm{MHz,} \mathrm{CDCl}\right): \delta 1.16(\mathrm{t}, J 7.6 \mathrm{~Hz}$, $3 \mathrm{H}), 2.13(\mathrm{~s}, 3 \mathrm{H}), 2.42(\mathrm{~s}, 3 \mathrm{H}), 2.43-2.56(\mathrm{~m}, 2 \mathrm{H}), 2.64(\mathrm{~s}, 3 \mathrm{H}), 2.68(\mathrm{~s}, 3 \mathrm{H}), 3.66(\mathrm{~d}, J 2.0 \mathrm{~Hz}, 1 \mathrm{H}), 4.87(\mathrm{~d}, J 2.0$ $\mathrm{Hz}, 1 \mathrm{H}), 5.85$ (d, J $2.2 \mathrm{~Hz}, 1 \mathrm{H}), 6.9$ ((dd, J 8.3, $2.2 \mathrm{~Hz}, 1 \mathrm{H}), 7.23-7.35(\mathrm{~m}, 3 \mathrm{H}), 7.55(\mathrm{~d}, J 8.3 \mathrm{~Hz}, 1 \mathrm{H}) ;{ }^{13} \mathrm{C} \mathrm{NMR}(125$ $\mathrm{MHz}_{\mathrm{CDCl}}$ ): $\delta 10.1,13.7,17.9,23.6,27.7,27.8,90.7,108.1,116.6,128.1,129.7,130.1,131.0,132.8,133.5$, 134.3, 136.3, 137.0, 137.1, 137.4, 141.9, 143.7, 157.0, 194.2, 199.0; MS (EI): $m / z$ (\%) 427 (46), 384 (100), 356 (96); HRMS (EI): $m / z$ calcd for $\mathrm{C}_{25} \mathrm{H}_{25} \mathrm{~N}_{5} \mathrm{O}_{2}: 427.2004$, found: 427.2008 .

1-\{1-[2-Isobutyryl-3-methylene-4-(2-ethyl-6-methylphenyl)-3,4-dihydroquinoxalin-6-yl]-5-methyl-1H-1,2,3triazol-4-yl\}-2-methylpropan-1-one (5j). Brown solid (111 mg, 46\%). mp 99-103 ${ }^{\circ} \mathrm{C} .{ }^{1} \mathrm{H} \mathrm{NMR}\left(600 \mathrm{MHz}, \mathrm{CDCl}_{3}\right)$ : $\delta 1.16(\mathrm{t}, J 7.6 \mathrm{~Hz}, 3 \mathrm{H}), 1.23(\mathrm{~d}, J 6.9 \mathrm{~Hz}, 6 \mathrm{H}), 1.24(\mathrm{~d}, J 6.9 \mathrm{~Hz}, 3 \mathrm{H}), 1.25$ (d, J $6.9 \mathrm{~Hz}, 3 \mathrm{H}), 2.13(\mathrm{~s}, 3 \mathrm{H}), 2.43(\mathrm{~s}$, $3 \mathrm{H}), 2.44-2.57(\mathrm{~m}, 2 \mathrm{H}), 3.61(\mathrm{~d}, J 2.0 \mathrm{~Hz}, 1 \mathrm{H}), 3.62-3.67(\mathrm{~m}, 2 \mathrm{H}), 4.47(\mathrm{~d}, J 2.0 \mathrm{~Hz}, 1 \mathrm{H}), 5.91(\mathrm{~d}, J 2.2 \mathrm{~Hz}, 1 \mathrm{H})$, $6.90(\mathrm{dd}, J 8.2,2.2 \mathrm{~Hz}, 1 \mathrm{H}), 7.24-7.36(\mathrm{~m}, 3 \mathrm{H}), 7.54(\mathrm{~d}, J 8.2 \mathrm{~Hz}, 1 \mathrm{H}) ;{ }^{13} \mathrm{C} \mathrm{NMR}\left(150 \mathrm{MHz}, \mathrm{CDCl}_{3}\right): \delta 10.1,13.7$, $17.5,17.6,17.8,18.6,23.6,26.9,37.1,37.4,90.0,108.1,116.7,128.1,129.7,130.1,130.5,132.9,133.6,135.2$,

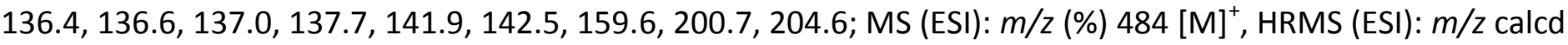
for $\mathrm{C}_{29} \mathrm{H}_{34} \mathrm{~N}_{5} \mathrm{O}_{2}: 484.2713$, found: 484.2711 .

Methyl 4-(2,6-dimethylphenyl)-6-fluoro-3-oxo-3,4-dihydroquinoxaline-2-carboxylate (6a). Beige precipitate (133 mg, 81\%). mp 134-135 ${ }^{\circ} \mathrm{C} .{ }^{1} \mathrm{H}$ NMR $\left(600 \mathrm{MHz}, \mathrm{CDCl}_{3}\right): \delta 1.99(\mathrm{~s}, 6 \mathrm{H}), 4.03(\mathrm{~s}, 3 \mathrm{H}), 6.25(\mathrm{dd}, J 9.7,2.5 \mathrm{~Hz}$, $1 \mathrm{H}), 7.11(\mathrm{dd}, J$ 8.7, $2.5 \mathrm{~Hz}, 1 \mathrm{H}), 7.26-7.29(\mathrm{~m}, 2 \mathrm{H}), 7.35-7.39(\mathrm{~m}, 1 \mathrm{H}), 8.02(\mathrm{dd}, J$ 8.7, $5.7 \mathrm{~Hz}, 1 \mathrm{H}) ;{ }^{13} \mathrm{C} \mathrm{NMR}(150$ $\mathrm{MHz}, \mathrm{CDCl}_{3}$ ): $\delta$ 17.6, 53.2, 101.2 (d, $J_{\mathrm{CF}} 28 \mathrm{~Hz}$ ), 112.9 (d, $J_{\mathrm{CF}} 24 \mathrm{~Hz}$ ), 128.7 (d, $J_{\mathrm{CF}} 2 \mathrm{~Hz}$ ), 129.4, 130.0, 132.8, 133.4 (d, $\left.J_{C F} 11 \mathrm{~Hz}\right), 135.2,135.7$ (d, $\left.J_{\mathrm{CF}} 12 \mathrm{~Hz}\right), 148.1$ (d, $\left.J_{\mathrm{CF}} 4 \mathrm{~Hz}\right), 151.1,163.6,165.1$ (d, $\left.J_{\mathrm{CP}} 255 \mathrm{~Hz}\right) ; \mathrm{MS}(\mathrm{ESI}): \mathrm{m} / z(\%)$ 327 [M] ${ }^{+}$HRMS (ESI): $m / z$ calcd for $\mathrm{C}_{18} \mathrm{H}_{16} \mathrm{FN}_{2} \mathrm{O}_{3}: 327.1145$, found: 327.1140 .

3-Benzoyl-1-(4-chlorophenyl)-7-fluoroquinoxalin-2(1H)-one (6b). Pale yellow crystals (113 mg, 60\%). mp 168$169{ }^{\circ} \mathrm{C} .{ }^{1} \mathrm{H}$ NMR $\left(600 \mathrm{MHz}, \mathrm{CDCl}_{3}\right): \delta 6.47$ (dd, J 9.8, 2.6 Hz, 1H), 7.10 (ddd, J 9.0, 8.0, 2.6 Hz, 1H), 7.28-7.31 (m, $2 \mathrm{H})$, 7.48-7.52 (m, 2H), 7.59-7.66 (m, 3H), $7.94\left(\mathrm{dd}, J\right.$ 9.0, 5.8 Hz, 1H), 8.01-8.04 (m, 2H); ${ }^{13} \mathrm{C} \mathrm{NMR}(150 \mathrm{MHz}$, $\left.\mathrm{CDCl}_{3}\right): \delta 102.3\left(\mathrm{~d}, J_{\mathrm{CP}} 28 \mathrm{~Hz}\right.$ ), 112.6 (d, $\left.J_{\mathrm{CP}} 24 \mathrm{~Hz}\right), 128.7,128.8,129.5,130.0,130.8,132.9\left(\mathrm{~d}, J_{\mathrm{CP}} 11 \mathrm{~Hz}\right), 133.0$, $134.3,134.7,136.1$ (d, $J_{\mathrm{CP}} 12 \mathrm{~Hz}$ ), 136.2, 152.6, 153.9 (d, $J_{\mathrm{CP}} 4 \mathrm{~Hz}$ ), 163.2, 164.2 (d, $J_{\mathrm{CP}} 254 \mathrm{~Hz}$ ), 190.9; MS (El): $m / z$ (\%) 380 (20), $378\left(41,[\mathrm{M}]^{+}\right), 349$ (21), 105 (100); HRMS (EI): $\mathrm{m} / z$ calcd for $\mathrm{C}_{21} \mathrm{H}_{12}{ }^{35} \mathrm{ClFN}_{2} \mathrm{O}_{2}: 378.0571$, found: 378.0572 .

Methyl 6-chloro-4-(4-methylphenyl)-3-oxo-3,4-dihydroquinoxaline-2-carboxylate (6c). Pale beige crystals (104 mg, 64\%). mp 206-207 ${ }^{\circ} \mathrm{C} .{ }^{1} \mathrm{H}$ NMR $\left(600 \mathrm{MHz}, \mathrm{CDCl}_{3}\right): \delta 2.48(\mathrm{~s}, 3 \mathrm{H}), 4.02(\mathrm{~s}, 3 \mathrm{H}), 6.74(\mathrm{~d}, J 2.1 \mathrm{~Hz}, 1 \mathrm{H})$, 7.14-7.17 (m, 2H), $7.31(\mathrm{dd}, J$ 8.6, $2.1 \mathrm{~Hz}, 1 \mathrm{H}), 7.41-7.44(\mathrm{~m}, 2 \mathrm{H}), 7.90(\mathrm{~d}, J 8.6 \mathrm{~Hz}, 1 \mathrm{H}) ;{ }^{13} \mathrm{C} \mathrm{NMR}(150 \mathrm{MHz}$, $\left.\mathrm{CDCl}_{3}\right): \delta 21.3,53.2,115.6,124.9,127.6,130.3,131.2,131.7,131.9,136.0,138.6,140.3,149.1,152.1,163.6$; MS (EI): $m / z$ (\%) 330 (38), 238 (71), 240 (100); HRMS (EI): $m / z$ calcd for $\mathrm{C}_{17} \mathrm{H}_{13}{ }^{35} \mathrm{CIN}_{2} \mathrm{O}_{3}$ : 328.0615, found: 328.0618.

Synthesis of 7-azidoquinazolinones 7a-c. General procedure. 7-Fluoro or 7-chloroquinoxalinone 6 (0.5 $\mathrm{mmol})$ and sodium azide $(162 \mathrm{mg}, 2.5 \mathrm{mmol})$ were stirred in DMF $(3.0 \mathrm{~mL})$ at room temperature $(6 \mathbf{a}$ and $6 \mathbf{b})$ or at 80 ${ }^{\circ} \mathrm{C}(6 \mathrm{c})$ for 12 - $48 \mathrm{~h}$ (tlc control). After the reaction was complete the mixture was dissolved with water and extracted with EtOAc. The extract was washed thoroughly with water then with brine and dried $\left(\mathrm{Na}_{2} \mathrm{SO}_{4}\right)$. 
After evaporation the solid residue was recrystallized from $i-\mathrm{PrOH}$ (7a and $7 \mathbf{b}$ ) or the product was isolated by column chromatography ( $\mathrm{SiO}_{2}$, hexane/EtOAc) (7c).

Methyl 6-azido-4-(2,6-dimethylphenyl)-3-oxo-3,4-dihydroquinoxaline-2-carboxylate (7a). Yellow powder (122 mg, 70\%). mp 153-155 ${ }^{\circ} \mathrm{C}(i-\mathrm{PrOH}) .{ }^{1} \mathrm{H} \mathrm{NMR}\left(600 \mathrm{MHz}, \mathrm{CDCl}_{3}\right): \delta 1.98(\mathrm{~s}, 6 \mathrm{H}), 4.03(\mathrm{~s}, 3 \mathrm{H}), 6.09(\mathrm{~d}, J 2.3 \mathrm{~Hz}$, $1 \mathrm{H}), 7.10(\mathrm{dd}, J$ 8.7, $2.3 \mathrm{~Hz}, 1 \mathrm{H}), 7.25-7.28(\mathrm{~m}, 2 \mathrm{H}), 7.34-7.38(\mathrm{~m}, 1 \mathrm{H}), 8.02(\mathrm{~d}, J 8.7 \mathrm{~Hz}, 1 \mathrm{H}) ;{ }^{13} \mathrm{C} \mathrm{NMR}(150 \mathrm{MHz}$, $\left.\mathrm{CDCl}_{3}\right): \delta 17.6,53.2,104.1,115.5,129.4,130.0,132.7,132.8,135.22,135.23,135.5,145.1,147.6,151.2,163.7$; MS (EI): m/z (\%) 349 (36), 321 (100), 306 (47), 274 (43), 234 (56); HRMS (EI): m/z calcd for $\mathrm{C}_{18} \mathrm{H}_{15} \mathrm{~N}_{5} \mathrm{O}_{3}$ : 349.1175, found: 349.1169 .

7-Azido-3-benzoyl-1-(4-chlorophenyl)quinoxalin-2(1H)-one (7b). Creamy powder (175 mg, 80\%). mp 172-176 ${ }^{\circ} \mathrm{C}$ (dec.); ${ }^{1} \mathrm{H}$ NMR $\left(600 \mathrm{MHz}, \mathrm{CDCl}_{3}\right): \delta 6.60$ (d, J $\left.2.2 \mathrm{~Hz}, 1 \mathrm{H}\right), 7.08$ (dd, J 8.6, $\left.2.2 \mathrm{~Hz}, 1 \mathrm{H}\right), 7.25-7.29(\mathrm{~m}, 2 \mathrm{H}), 7.48-$ $7.52(\mathrm{~m}, 2 \mathrm{H}), 7.59-7.66(\mathrm{~m}, 3 \mathrm{H}), 7.93(\mathrm{~d}, \mathrm{~J} 8.6 \mathrm{~Hz}, 1 \mathrm{H}), 8.01-8.04(\mathrm{~m}, 1 \mathrm{H}) ;{ }^{13} \mathrm{C} \mathrm{NMR}\left(150 \mathrm{MHz}, \mathrm{CDCl}_{3}\right): \delta 105.3$, 115.3, 128.7, 129.3, 129.6, 130.1, 130.8, 132.4, 132.9, 134.3, 134.8, 136.0, 136.1, 144.0, 152.7, 153.7, 191.0; MS (EI): m/z (\%) 401 (7), 375 (28), 373 (33), 345 (33), 105 (100); HRMS (EI): m/z calcd for $\mathrm{C}_{21} \mathrm{H}_{12}{ }^{35} \mathrm{CIN}_{5} \mathrm{O}_{2}$ : 401.0680, found: 401.0682 .

Methyl 6-azido-4-(4-methylphenyl)-3-oxo-3,4-dihydroquinoxaline-2-carboxylate (7c). Yellow crystals (62 mg, 37\%). mp $180{ }^{\circ} \mathrm{C}$ (dec.); ${ }^{1} \mathrm{H}$ NMR (500 MHz, CDCl $): \delta 2.47(\mathrm{~s}, 3 \mathrm{H}), 3.17(\mathrm{~s}, 3 \mathrm{H}), 6.29(\mathrm{~d}, J 2.3 \mathrm{~Hz}, 1 \mathrm{H}), 7.05$ (dd, J 8.6, 2.3 Hz, 1H), 7.13-7.16 (m, 2H), 7.39-7.42 (m, 2H), $7.96(\mathrm{~d}, J 8.6 \mathrm{~Hz}, 1 \mathrm{H}) ;{ }^{13} \mathrm{C} \mathrm{NMR}\left(150 \mathrm{MHz}, \mathrm{CDCl}_{3}\right): \delta 21.3$, $53.2,105.5,115.3,127.6,129.2,131.1,131.9,132.5,136.8,140.2,144.4,147.5,152.3,163.7 ; \mathrm{MS}$ (EI): $\mathrm{m} / z$ (\%) 335 (45), 307 (100), 279 (51), 247 (59), 219 (75), 304 (71); HRMS (EI): m/z calcd for $\mathrm{C}_{17} \mathrm{H}_{13} \mathrm{~N}_{5} \mathrm{O}_{3}: 335.1018$ ， found: 335.1027

Synthesis of triazoles $8 \mathrm{a}-\mathrm{c}$ from dihydroquinoxalines $\mathbf{7 a - c}$. A solution of $7(0.5 \mathrm{mmol})$, acetylacetone (1.1 mmol) and $t-\mathrm{BuNH}_{2}(175 \mathrm{mg}, 2.4 \mathrm{mmol})$ in $\mathrm{CH}_{3} \mathrm{CN}(5.0 \mathrm{~mL})$ was stirred for $24 \mathrm{~h}$ at room temperature. The volatile materials were evaporated and the residue was. The crude product was purified by column chromatography $\left(\mathrm{SiO}_{2}\right.$, hexane/ethyl acetate) (8a) or treated with $\mathrm{MeOH}$ and the precipitate was filtered off, washed with $\mathrm{MeOH}$ and dried on air (8b and $\mathbf{8 c}$ ). Analytical samples were recrystallized from $\mathrm{MeOH}$ or EtOAc.

Methyl 6-(4-acetyl-5-methyl-1H-1,2,3-triazol-1-yl)-4-(2,6-dimethylphenyl)-3-oxo-3,4-dihydroquinoxaline-2carboxylate (8a). Pale yellow powder (100 mg, 46\%). mp 218-219 ${ }^{\circ} \mathrm{C}(\mathrm{MeOH}) .{ }^{1} \mathrm{H} \mathrm{NMR}\left(500 \mathrm{MHz}^{\mathrm{C}} \mathrm{CDCl}\right): \delta$ $2.02(\mathrm{~s}, 6 \mathrm{H}), 2.50(\mathrm{~s}, 3 \mathrm{H}), 2.70(\mathrm{~s}, 3 \mathrm{H}), 4.06(\mathrm{~s}, 3 \mathrm{H}), 6.65(\mathrm{~d}, J 2.0 \mathrm{~Hz}, 1 \mathrm{H}), 7.25-7.28(\mathrm{~m}, 2 \mathrm{H}), 7.33-7.38(\mathrm{~m}, 1 \mathrm{H})$, $7.52(\mathrm{dd}, J$ 8.6, $1 \mathrm{H}), 8.22$ (d, J $8.6 \mathrm{~Hz}, 1 \mathrm{H}) ;{ }^{13} \mathrm{C} \mathrm{NMR}\left(125 \mathrm{MHz}, \mathrm{CDCl}_{3}\right): \delta$ 10.2, 17.6, 27.9, 53.4, 110.8, 120.6, 129.6, 130.4, 132.0, 132.3, 132.6, 134.7, 135.2, 137.2, 138.2, 144.0, 150.8, 151.3, 163.4, 194.1; MS (EI): $\mathrm{m} / \mathrm{z}$ (\%) 431 (94), 403 (61), 388 (56), 361 (46), 316 (59), 301 (60), 272 (68), 219 (100); HRMS (El): m/z calcd for $\mathrm{C}_{23} \mathrm{H}_{12} \mathrm{~N}_{5} \mathrm{O}_{4}: 431.1594$, found: 431.1602 .

7-(4-Acetyl-5-methyl-1H-1,2,3-triazol-1-yl)- 3-benzoyl-1-(4-chlorophenyl)quinoxalin-2(1H)-one (8b). Pale yellow crystals (200 mg, 81\%). mp 246-247 ${ }^{\circ} \mathrm{C}$ (EtOAc). ${ }^{1} \mathrm{H} \mathrm{NMR}\left(500 \mathrm{MHz}, \mathrm{CDCl}_{3}\right): \delta 2.59(\mathrm{~s}, 3 \mathrm{H}), 2.74(\mathrm{~s}, 3 \mathrm{H})$, $6.95(\mathrm{~d}, J 2.0 \mathrm{~Hz}, 1 \mathrm{H}), 7.33-7.36(\mathrm{~m}, 2 \mathrm{H}), 7.48(\mathrm{dd}, J$ 8.6, 2.0 Hz, 1H), $7.55(\mathrm{t}, J 7.7 \mathrm{~Hz}, 2 \mathrm{H}), 7.59-7.63(\mathrm{~m}, 2 \mathrm{H})$, 7.67-7.71 (m, 1H), $8.06(\mathrm{~d}, J 7.5 \mathrm{~Hz}, 2 \mathrm{H}), 8.17(\mathrm{~d}, J 8.6 \mathrm{~Hz}, 1 \mathrm{H}) ;{ }^{13} \mathrm{C} \mathrm{NMR}\left(125 \mathrm{MHz}, \mathrm{CDCl}_{3}\right): \delta 10.3,27.9,112.3$, $120.5,128.8,129.5,130.0,131.0,132.1,132.2,132.5,134.4,134.6,135.4,136.6,137.3,137.4,144.0,152.3$, 156.9, 190.5, 194.1; MS (EI): $m / z$ (\%) 483 (3), 413 (12), 105 (100); HRMS (EI): $\mathrm{m} / z$ calcd for $\mathrm{C}_{26} \mathrm{H}_{18}{ }^{35} \mathrm{CIN}_{5} \mathrm{O}_{3}$ : 483.1098, found: 483.1107.

Methyl 6-(4-acetyl-5-methyl-1H-1,2,3-triazol-1-yl)-4-(4-methylphenyl)-3-oxo-3,4-dihydroquinoxaline-2carboxylate (8c). Colorless powder (81 mg, 81\%). mp 255-257 ${ }^{\circ} \mathrm{C}$ (i-PrOH/EtOAc). ${ }^{1} \mathrm{H} \mathrm{NMR}\left(500 \mathrm{MHz} \mathrm{CDCl}_{3}\right): \delta$ $2.45(\mathrm{~s}, 3 \mathrm{H}), 2.53(\mathrm{~s}, 3 \mathrm{H}), 2.71(\mathrm{~s}, 3 \mathrm{H}), 4.05$ (s, 3H), 6.87 (d, J $2.1 \mathrm{~Hz}, 1 \mathrm{H}), 7.17-7.20(\mathrm{~m}, 2 \mathrm{H}), 7.39-7.42$ (, 2H), 
7.45 (dd, J 8.5, $2.1 \mathrm{~Hz}, 1 \mathrm{H}), 8.17$ (d, J $8.5 \mathrm{~Hz}, 1 \mathrm{H}) ;{ }^{13} \mathrm{C} \mathrm{NMR}\left(125 \mathrm{MHz}, \mathrm{CDCl}_{3}\right): \delta$ 10.3, 21.3, 27.9, 53.4, 112.4, $120.3,127.5,131.3,131.4,131.8,132.3,136.1,137.3,137.6,140.6,143.9,151.0,151.9,163.4,194.1 ; M S$ (EI): m/z (\%) 417 (56), 389 (70), 374 (47), 347 (100), 260 (50); HRMS (El): m/z calcd for $\mathrm{C}_{22} \mathrm{H}_{19} \mathrm{~N}_{5} \mathrm{O}_{4}:$ 417.1437, found: 417.1433 .

\section{Acknowledgements}

The authors thank Dr. Bogdan K. Wilk from Monmouth University, West Long Branch, NJ, USA for his vital help in the preparation of the manuscript.

\section{References}

1. Bonandi, E.; Christodoulou, M. S.; Fumagalli, G.; Perdicchia, D.; Rastelli, G.; Passarella, D. Drug Discovery Today 2017, 22, 1572.

https://doi.org/10.1016/i.drudis.2017.05.014

2. Zhou C. -H.; Wang Y. Curr. Med. Chem. 2012, 19, 239.

https://doi.org/10.2174/092986712803414213

3. Kharb, R,; Sharma, P. C.; Yar, M. S. J. Enzyme Inhib. Med. Chem. 2011, 26, 1. https://doi.org/10.3109/14756360903524304

4. Haider, S.; Alam, M. S.; Hamid, H. Inflammation \& Cell Signaling 2014, 1, e95.

5. Livermore, D. G. H.; Bethell, R. C.; Cammack, N.; Hancock, A. P.; Hann, M. M.; Green, D. V. S.; Lamont, R. B.; Noble, S. A.; Orr, D. C.; Payne, J. J.; Ramsay, M. V. J.; Shingler, A. H.; Smith, C.; Storer, R.; Williamson, Ch.; Willson, T. J. Med. Chem. 1993, 36, 3784. https://doi.org/10.1021/jm00076a005

6. Hennequin, L. F.; Thomas, A. P.; Johnstone, C.; Stokes, E. S. E.; Plé, P. A.; Lohmann, J.-J. M.; Ogilvie, D. J.; Dukes, M.; Wedge, S. R.; Curwen, J. O.; Kendrew, J.; Lambert-van der Brempt, Ch. J. Med. Chem. 1999, 42, 5369.

https://doi.org/10.1021/jm990345w

7. Wróbel, Z.; Kwast, A. Synlett 2007, 1525. https://doi.org/10.1055/s-2007-982534

8. Wróbel, Z.; Kwast, A. Synthesis 2010, 3865. https://doi.org/10.1055/s-0030-1258230

9. Kwast, A.; Stachowska, K.; Trawczyński, A.; Wróbel, Z. Tetrahedron Lett. 2011, 6484. https://doi.org/10.1016/i.tetlet.2011.09.113

10. Wróbel, Z.; Stachowska, K.; Kwast, A. Eur. J. Org. Chem. 2014, 7721.

https://doi.org/10.1002/ejoc.201402624

11. Wróbel, Z.; Stachowska, K.; Grudzień, K.; Kwast, A. Synlett 2011, 1439. https://doi.org/10.1055/s-0030-1260764

12. Wróbel, Z.; Stachowska, K.; Kwast, A.; Gościk, A.; Królikiewicz, M.; Pawłowski, R.; Turska, I. Helv. Chim. Acta 2013, 96, 956. 
13. Wróbel, Z.; Stachowska, K.; Kwast, A. Synthesis 2013, 45, 127.

https://doi.org/10.1055/s-0032-1316823

14. Boulton, A. J.; Ghosh, P. B.; Katritzky, A. R. J. Chem. Soc.(B) 1966, 1004.

https://doi.org/10.1039/j29660001004

15. Gornostaev, L. M.; Bocharova, E. A.; Dolgushina, L. V.; Begryanskaya, L. Yu.; Gatilov, Yu. V. Russ. J. Org. Chem. 2010, 46, 693.

https://doi.org/10.1134/S1070428010050179

16. Meldal, M.; Tornøe, C. W. Chem. Rev. 2008, 108, 2952.

https://doi.org/10.1021/cr0783479

17. Trawczyński, A.; Wróbel, Z. Synlett 2014, 25, 2773.

https://doi.org/10.1055/s-0034-1379238

18. Trawczyński, A.; Telega, M.; Wróbel, Z. Synlett 2015, 26, 1352.

https://doi.org/10.1055/s-0034-1380515

19. Zeghada, S.; Bentabed-Ababsa, G.; Derdour, A.; Abdelmounim, S.; Domingo, L. T.; Sáez, J. A.; Roisnel, T.; Nassar, E.; Mongin, F. Org. Biomol. Chem. 2011, 9, 4295.

https://doi.org/10.1039/c1ob05176h

20. Singh, H.; Sindhu, J.; Khurana, J. M. RSC Advances 2013, 3, 22360.

https://doi.org/10.1039/c3ra44440f

21. Danence, L. J. T.; Gao, Y.; Li, M.; Huang, Y.; Wang, J. Chem. Eur. J. 2011, 17, 3584. https://doi.org/10.1002/chem.201002775

22. In the ${ }^{13} \mathrm{C}$ NMR spectra of 2-nitrosoanilines two carbon atoms ortho to the nitroso group often give very broad, thus exceptionally low, signals which are difficult to observe in the standard spectra recorded at ambient temperature. This issue has been observed also by others and reported in the literature, e.g. ref 23.

23. Lipilin, D. L.; Churakov, A. M.; Ioffe, S. L.; Strelenko, Y. A.; Tartakovsky, V. A. Eur. J. Org. Chem. $1999,29$. https://doi.org/10.1002/(SICI)1099-0690(199901)1999:1<29::AID-EJOC29>3.0.CO;2-M 